\title{
BMJ Open Surgery and COVID-19: a rapid scoping review of the impact of the first wave of COVID-19 on surgical services
}

To cite: 0 'Rielly C, $\mathrm{Ng}$ Kamstra J, Kania-Richmond A, et al. Surgery and COVID-19: a rapid scoping review of the impact of the first wave of COVID-19 on surgical services. BMJ Open 2021;11:e043966. doi:10.1136/ bmjopen-2020-043966

- Prepublication history and additional supplemental material for this paper are available online. To view these files, please visit the journal online (http://dx.doi.org/10.1136/ bmjopen-2020-043966)

Received 22 August 2020 Accepted 18 May 2021

Check for updates

(C) Author(s) (or their employer(s)) 2021. Re-use permitted under CC BY-NC. No commercial re-use. See rights and permissions. Published by BMJ.

For numbered affiliations see end of article.

Correspondence to

Dr Khara Sauro;

kmsauro@ucalgary.ca

\section{ABSTRACT}

Objectives To understand how surgical services have been reorganised during and following public health emergencies, particularly the first wave of the COVID-19 pandemic, and the consequences for patients, healthcare providers and healthcare systems.

Design A rapid scoping review.

Setting We searched the MEDLINE, Embase and grey literature sources for documents and press releases from governments and surgical organisations or associations.

Participants Studies examining surgical service delivery during public health emergencies including COVID-19, and the impact on patients, providers and healthcare systems were included.

Primary and secondary outcome measures Primary outcomes were strategies implemented for the reorganisation of surgical services. Secondary were the impacts of reorganisation and resuming surgical services, such as: adverse events (including morbidity and mortality), primary care and emergency department visits, length of hospital and ICU stay, and changes to surgical waitlists.

Results One hundred and thirty-two studies were included in this review; 111 described reorganisation of surgical services, 55 described the consequences of reorganising surgical services; and 6 reported actions taken to rebuild surgical capacity in public health emergencies. Reorganisations of surgical services were grouped under six domains: case selection/ triage, personal protective equipment (PPE) regulations and practice, workforce composition and deployment, outpatient and inpatient patient care, resident and fellow education, and the hospital or clinical environment. Service reorganisations led to large reductions in non-urgent surgical volumes, increases in surgical wait times and impacted medical training (ie, reduced case involvement) and patient outcomes (eg, increases in pain). Strategies for rebuilding surgical capacity were scarce but focused on the availability of staff, PPE and patient readiness for surgery as key factors to consider before resuming services.

Conclusions Reorganisation of surgical services in response to public health emergencies appears to be context dependent and has far-reaching consequences that must be better understood in order to optimise future health system responses to public health emergencies.

\section{Strengths and limitations of this study}

This rapid scoping review provides an exhaustive and rigorous summary of the academic and grey literature regarding modifications to surgical services in response to public health emergencies, especially the first wave of COVID-19.

- This study did not limit studies based on location or language of publication to ensure contributions from worldwide voices in the context of a worldwide pandemic.

Both quantitative and qualitative outcomes were included, with a mix of inductive and deductive data abstraction approaches to provide a comprehensive understanding of surgical services during public health emergencies.

- Studies with potential relevance to this question are emerging at an unprecedented rate in response to the COVID-19 pandemic, and as such, some may not be included in the current review.

\section{INTRODUCTION}

The novel SARS-CoV-2 (COVID-19) virus has spread across the globe with unrelenting speed. At the time of writing, over 88 million cases have been confirmed with 1.9 million fatalities. ${ }^{1}$ To protect the most vulnerable in our societies, efforts to curb further escalation (eg, travel restrictions and physical distancing) have had a focal objective: to prevent surges that could overwhelm healthcare including shortages in personal protective equipment (PPE), ventilators and hospital capacity.

Medical institutions have taken steps to maximise staff, PPE, ventilators and intensive care unit (ICU) capacity in case public health efforts to 'flatten the curve' are insufficient. Most notably, surgical programmes have suspended non-urgent (or 'elective') surgical procedures. Non-urgent surgeries are often defined as procedures for which a delay of 3 months or longer would not result in significant adverse effects to the patient. ${ }^{23}$ These changes have thrust patients, providers 
and healthcare organisations into previously unexplored territory.

While governing bodies such as colleges and academies of surgery have made recommendations to alter surgical service delivery in response to COVID-19, they have not always provided explicit instructions on how programmes should operationalise the recommendations. As such, approaches to surgical triage and service delivery remain unclear: who has done what, where and why? Furthermore, the impacts of adopting these recommendations on surgical programmes, and more importantly, the physical and psychological well-being of patients and healthcare providers have only been hypothesised. ${ }^{4}$ Lastly, once COVID-19 begins to release its grip on the world and the postpandemic recovery begins, programmes will be tasked with rebuilding the surgical capacity necessary to reschedule and tackle the backlog of postponed procedures. Evidence distilled from the experiences of others in the context of COVID-19 and other public health emergencies (ie, H1N1, Ebola and SARS) is needed to guide approaches to surgical service delivery.

To enable evidence-informed reorganisation and resumption of non-urgent surgeries during COVID-19 and for future public health emergencies, we conducted a rapid scoping review to identify and map the available literature. Our objective was to understand how surgical services have been reorganised during and following public health emergencies, particularly the first wave of the COVID-19 pandemic, and the consequences of these changes for patients, healthcare providers and healthcare systems.

\section{METHODS}

\section{Study design}

This scoping review followed the Joanna Briggs Institute methodology and Preferred Reporting Items for Systematic Reviews and Meta-Analyses extension for Scoping Reviews checklist. ${ }^{56}$ The rapidly evolving situation of the current COVID-19 pandemic demanded a similarly rapid evidence synthesis. Therefore, methodological concessions recommended by the WHO and Cochrane guidance for rapid reviews were made. ${ }^{78}$ Specifically, following a pilot exercise involving triplicate review and consensus for 50 abstracts only a $25 \%$ random sample of the remaining abstracts were reviewed in duplicate. Furthermore, while language limitations were not applied to the search, manuscripts not written in English that could not be translated by members of the research team were not eligible for data extraction, although their references were still included. This review addressed three research questions to achieve our objective: (1) how have surgical services been reorganised in response to public health emergencies, especially the first wave of the COVID-19 pandemic?; (2) what are the patient-level, healthcare provider-level and system-level consequences of reorganising surgical services?; and (3) what approaches have been used for resuming surgical services?

\section{Search strategy}

The search strategy was developed by two investigators (CO and KS) and refined by others with context expertise in surgery and literature review methodology (JN-K and AK-R). The search strategy included subject headings, keywords and synonyms identifying public health emergencies in general and specific public health emergencies (Ebola, SARS-CoV1, H1N1 and MERS) and surgery and as tailored for each database (online supplemental appendix A). Given the exploratory nature of the review, we did not filter by study design or publication type, and since the impacts of a pandemic spans many countries, there were no language restrictions.

We used the search strategy to search MEDLINE (including Epub Ahead of Print, In-Process \& Other NonIndexed Citations) and Embase from inception until 8 May 2020. Anticipating pertinent information may not be published (ie, joint statements, recommendations and guidelines from surgical colleges); we supplemented the database search with a structured grey literature search including targeted website searching, advanced and general Google searching and contact with knowledge experts (online supplemental appendix A). ${ }^{9}$ The reference lists of included studies were screened for relevant studies not otherwise captured.

\section{Study selection}

Titles and abstracts were reviewed by one of two independent reviewers with a third independent reviewer screening $25 \%$ of randomly selected references in duplicate. Full texts of studies considered potentially eligible at title/abstract screening phase by at least one reviewer were reviewed in duplicate by two reviewers for eligibility. Any disagreement between reviewers at the full-text screening phase was resolved through discussion and did not necessitate a third reviewer. If studies were excluded at the full-text screening phase, the reason for exclusion was noted. Fulltext articles meeting eligibility criteria were included, and data were abstracted using a standardised data abstraction form (online supplemental appendix B). At both stages of screening, a pilot sample of 50 articles were jointly reviewed by both reviewers to ensure reliable application of eligibility criteria between reviewers.

\section{Study eligibility}

Studies were eligible for inclusion if they discussed alterations to surgical services during public health emergencies and reported: (1) reorganisation of surgical services, (2) impact of reorganising surgical services on patients, healthcare providers or healthcare system or (3) approaches to resuming surgical capacity. Studies of any design or publication date were eligible for inclusion. Studies in any language were eligible, but consistent with rapid review methods, studies not easily translated by authors were excluded from the data synthesis, although citations are still provided. Studies were excluded if they described: only urgent interventions arising during a hospital admission (eg, emergency tracheostomy and 
caesarean section), settings beyond in-patient acute care (eg, outpatient clinics including dental clinics), changes to surgical service delivery not made in direct response to a public health emergency and healthcare services not specifically related to surgical service.

Notably, our intention was to include guidelines that made recommendations regarding provision of surgical services; however, a high-quality review of guidelines was published ${ }^{10}$ during the preparation of this review and as such, we chose to exclude guidelines.

\section{Data extraction}

Data were abstracted by one reviewer and verified by a second reviewer, using a standardised data abstraction form (online supplemental appendix B). Data included: date of publication, country, study design, definition of non-urgent surgery, characteristics of study sample (if applicable) and outcomes of interest for the three research questions, detailed further.

\section{Outcomes of interest}

Our primary outcomes were reorganisation of surgical services, impact of reorganisation and resuming surgical services. We intentionally included a broad array of outcomes and used an inductive approach to data abstraction to gain a comprehensive understanding of surgical services and the impact during public health emergencies.

We collected qualitative data from studies reporting on changes to surgical programming, conceptualised into five categories: changes to triage criteria or case selection, changes to PPE practices, workforce changes, changes to patient care, changes to resident and fellow education and environmental changes. Qualitative and quantitative data on the impact of reorganisation of surgical services was organised by impact on: patients, providers and healthcare system. To illustrate temporal changes, data preceding, during and after the precipitating event, were collected whenever possible. Quantitative variables of interest included: adverse events (including morbidity and mortality), primary care and emergency department visits, number of hospital and ICU admissions, length of hospital and ICU stay, number of surgical procedures performed and number of procedures cancelled, care costs and wait times for non-urgent surgery. Qualitative variables included narrative description of patient or physician experience, written descriptions of changes to physician remuneration or comments surrounding surgical waitlist composition. Qualitative data were also collected on details of efforts to rebuild capacity to surgical services.

\section{Study quality (risk of bias) assessment}

Given the aim of a rapid scoping review is not to appraise evidence but to map the available literature, ${ }^{11}$ quality appraisal of included studies was not performed.

\section{Data synthesis, analysis and reporting}

Consistent with our objectives and scoping review methodology, ${ }^{12}$ we did not to perform quantitative analysis but did use descriptive statistics to summarise quantitative outcomes. We characterised and mapped the available emerging evidence using an inductive qualitative approach. Specifically, two authors (CO and KS) familiarised themselves with the included studies and, throughout the data extraction process, continuously identified and specified recurrent categories emerging from the data. This was a non-linear process that continued until both authors were satisfied that the selected categories represented all important aspects of the evidence. The final categories are presented. Data were synthesised and presented separately for each of the three research questions.

\section{Patient and public involvement}

Patients and the public were not involved in study design, execution or interpretation.

\section{Statement of ethics approval}

Ethics approval was not required because this study did not involve humans or animals, but rather only included published data.

\section{RESULTS}

\section{Search results}

A total of 3013 unique scholarly articles and 106 sources of grey literature were identified, of which 702 were considered eligible for full-text review. After full-text review, 120 studies and five documents from the grey literature were included. Screening of the reference lists of included articles led to seven additional studies being included for a total of 132 included studies. Thirtyseven studies contributed data to more than one of the research questions resulting in the qualitative synthesis of 111 studies assessing alterations to service delivery, 55 studies evaluating the consequences of these changes and 6 studies enumerating their procedures for rebuilding capacity (table 1). The flow of evidence sources within the study is detailed in figure 1. One Spanish language study was translated for inclusion, ${ }^{13}$ but two studies could not be readily translated; therefore, they are not included in the synthesis. ${ }^{1415}$

\section{Description of studies}

The majority of included studies were published in 2020 about COVID-19 (87.9\%, n=116); fewer studies were related to other public health emergencies: SARS (7.58\%, $\mathrm{n}=10)$, Ebola (2.27\%, $\mathrm{n}=3)$, H1N1 $(1.52 \%, \mathrm{n}=2)$ and MERS $(0.76 \%, \mathrm{n}=1)$. Over two-thirds of the included studies $(74.2 \%)$ emerged from the countries hit earliest by COVID-19: China $(14.4 \%, \mathrm{n}=19)$, Singapore $(8.33 \%$, $\mathrm{n}=11)$, Italy $(19.7 \%, \mathrm{n}=26)$ and the USA $(31.8 \%, \mathrm{n}=41)$. While many studies described the experiences of their surgical departments as a whole, oncology $(15.9 \%, n=21)$, orthopaedics $(13.6 \%, \mathrm{n}=18)$ and neurosurgery $(11.4 \%$, $\mathrm{n}=15$ ) were the specialties most prominently represented. Summaries of descriptive study information are shown in figure 2. 


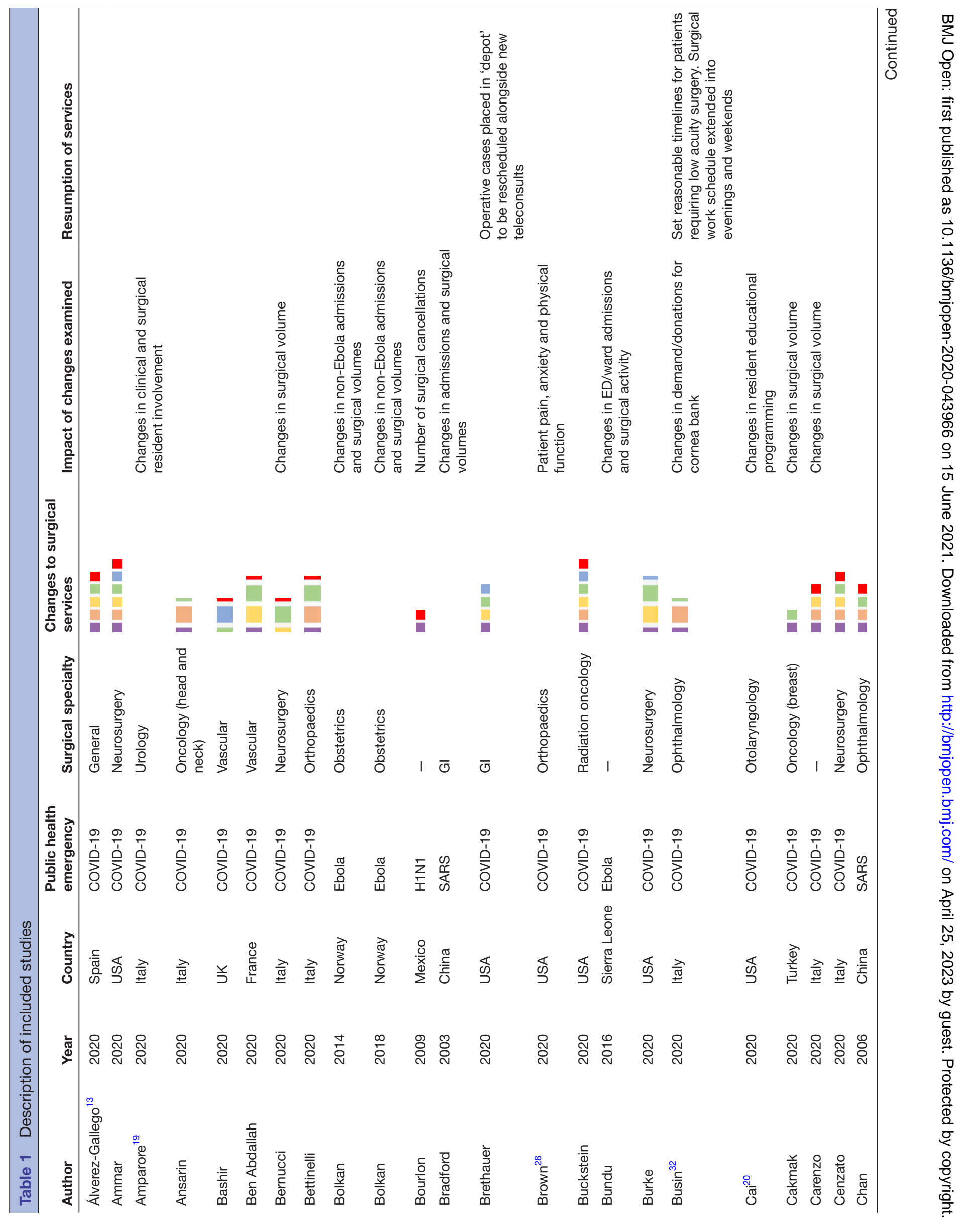




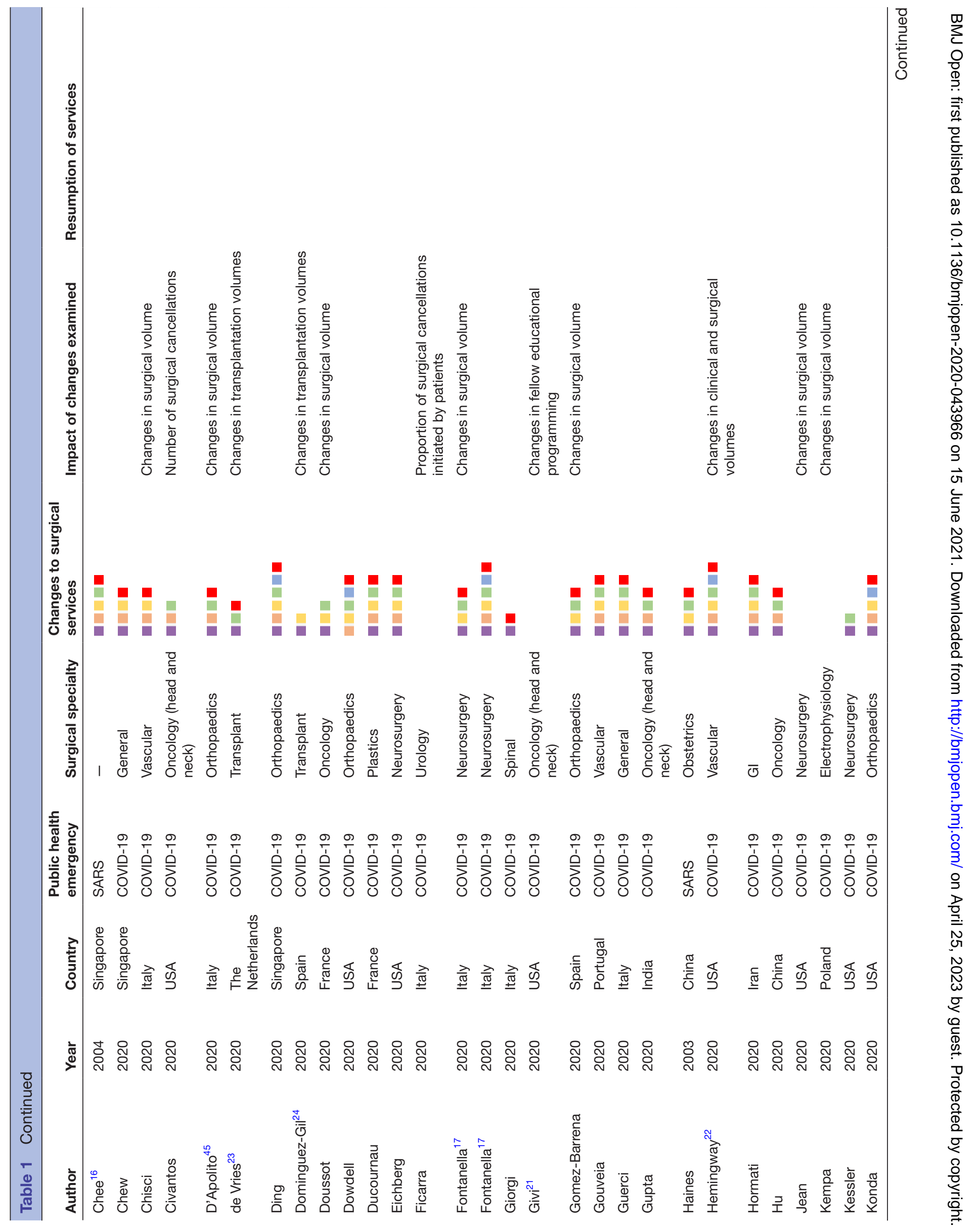




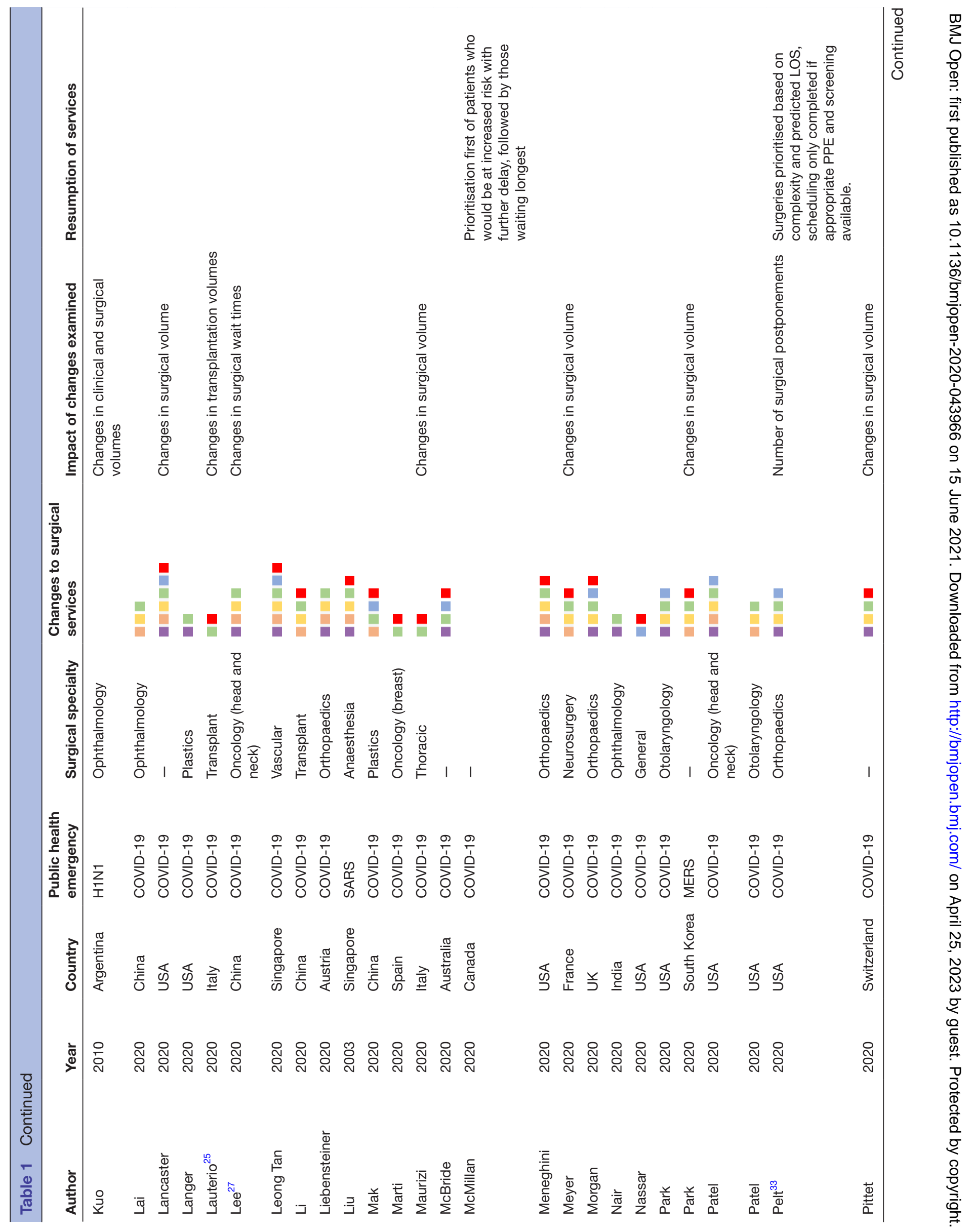




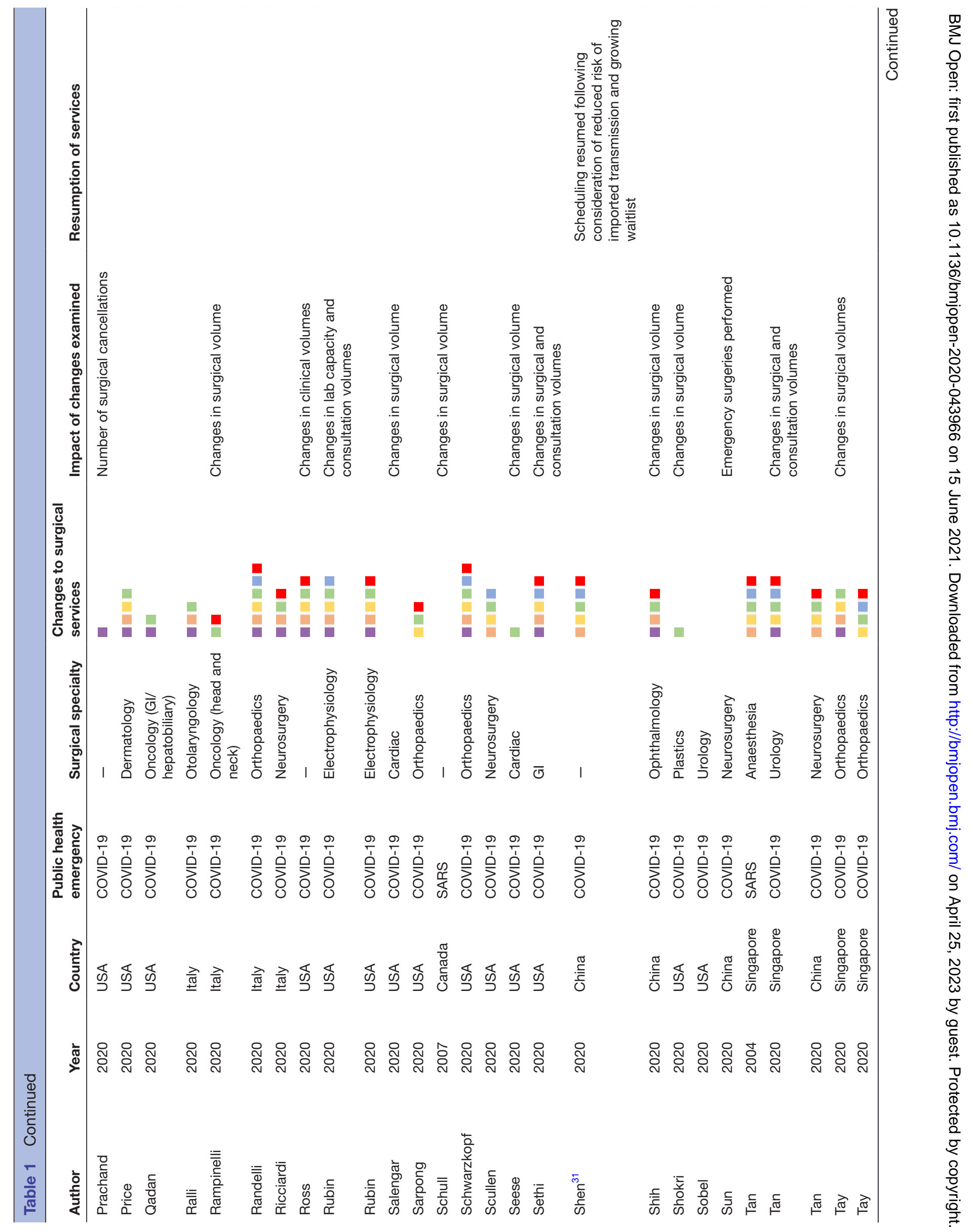




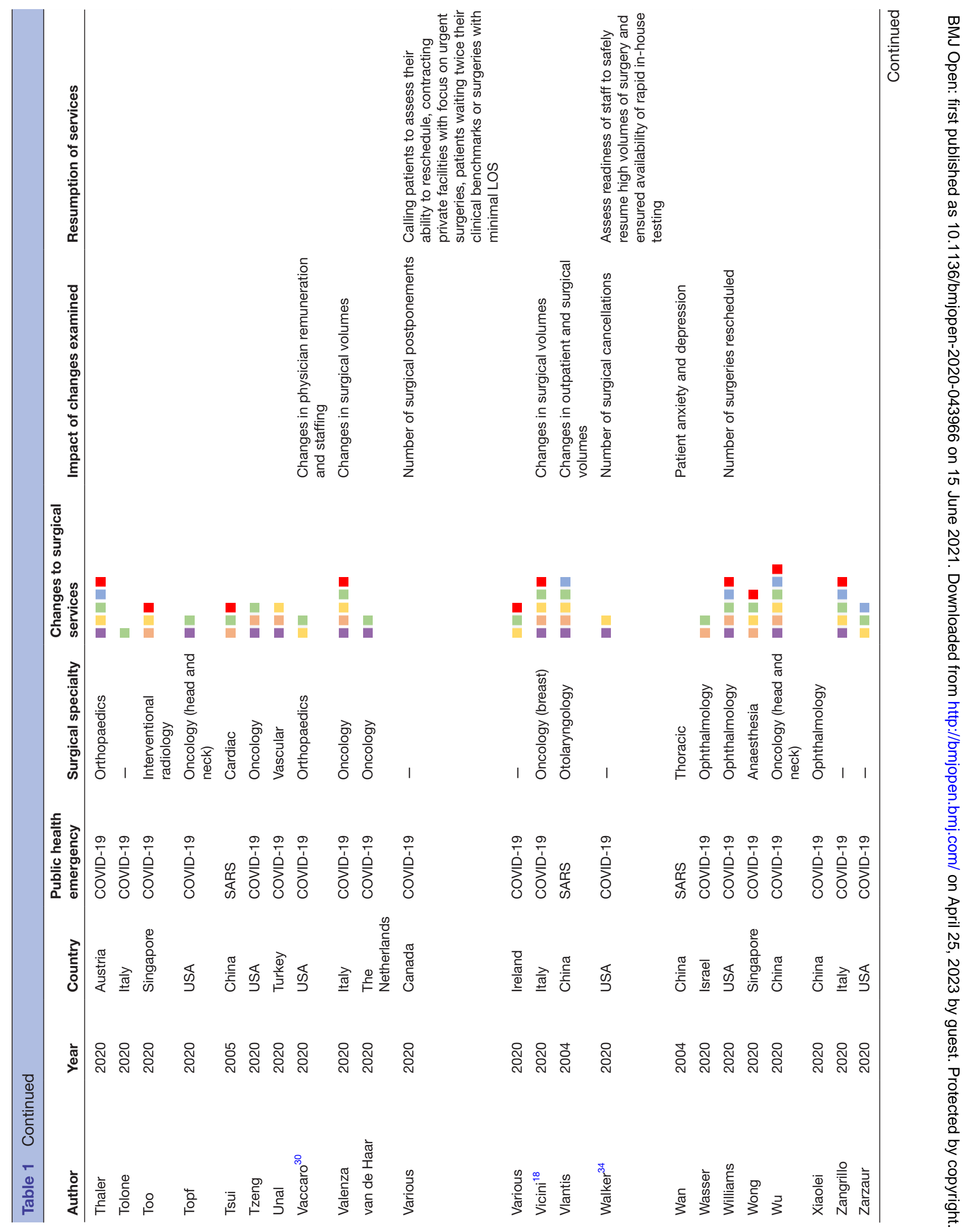




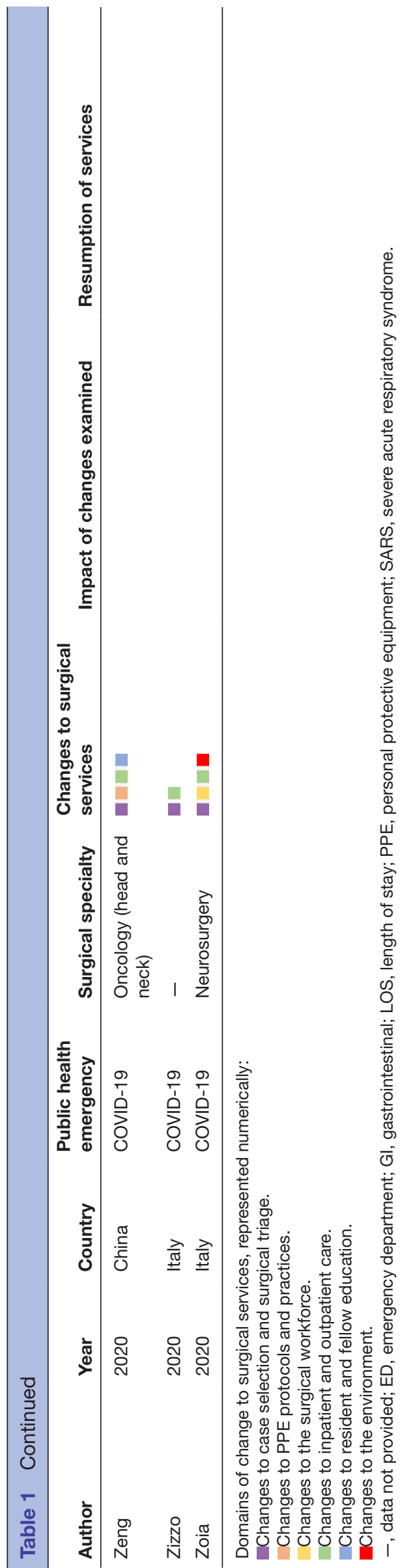

\section{Reorganisation of surgical service}

A number of categories emerged from the 108 studies describing reorganisation of surgical services. Nearly all studies reported partial, with most reporting full cessation of non-urgent surgeries at their centre, although with varying definitions of 'non-urgent' (eg, can be safely postponed for 3 months) and 'urgent' (eg, patient would have adverse outcome if not completed within 7 days). Changes to service delivery were focused on six domains: case selection/triage, PPE regulations and practice, workforce composition and deployment, outpatient and inpatient patient care, resident and fellow education, and the hospital or clinical environment (table 2). The three domains that were most frequently reported (case selection/triage, patient care and workforce) are described in greater detail below.

Changes to case selection and triage procedures. The countries and surgical specialties most effected by pandemicrelated changes to service delivery are described above; however, the issue of which patients can safely undergo what surgical procedures was also discussed in the included studies. We identified cancelling or postponing 'non-urgent' surgeries was almost universal. Most often hospitals cancelled surgeries via telephone or text message, but some studies identified that patients initiated their own surgical cancellations due to concerns with safety and nosocomial infection. While urgent surgeries were triaged according to routine practice, new triage decisions were made for non-urgent (including oncology) procedures. Methods for triaging non-urgent procedures varied across studies, from the use of guideline supported checklists of eligible procedures to virtual multidisciplinary meetings where the treating surgeon presented details of the case (eg, patient characteristics, acuity and imaging) to a larger group representing many surgical specialties to reach consensus on each case.

Changes to patient care. Sixty-two studies reported complete cessation or marked reduction of in-person, non-urgent outpatient clinic visits. In these studies, only urgent patients and those requiring postoperative suture or staple removal were granted in-person visits under strict conditions including mask wearing, negative symptom check, history or temperature prescreening. Studies specific to COVID-19 almost universally filled the resulting care gap for patients deemed 'non-urgent' using telephone or video-based telemedicine. Interfaces used include, but were not limited to, Zoom, WeChat, Facetime, telephone and SMS text messaging. A reported advantage of telemedicine was the ability to follow-up with returning patients and to continue consultations and establish contact with new patients who would require care when non-urgent surgeries resumed. While some admitted a historical reluctance to transition to video-based telemedicine and reported early concerns with their ability to establish secure connections with patients, frequently their worries faded with use and many reported telemedicine would remain integrated in their practices beyond the pandemic. 


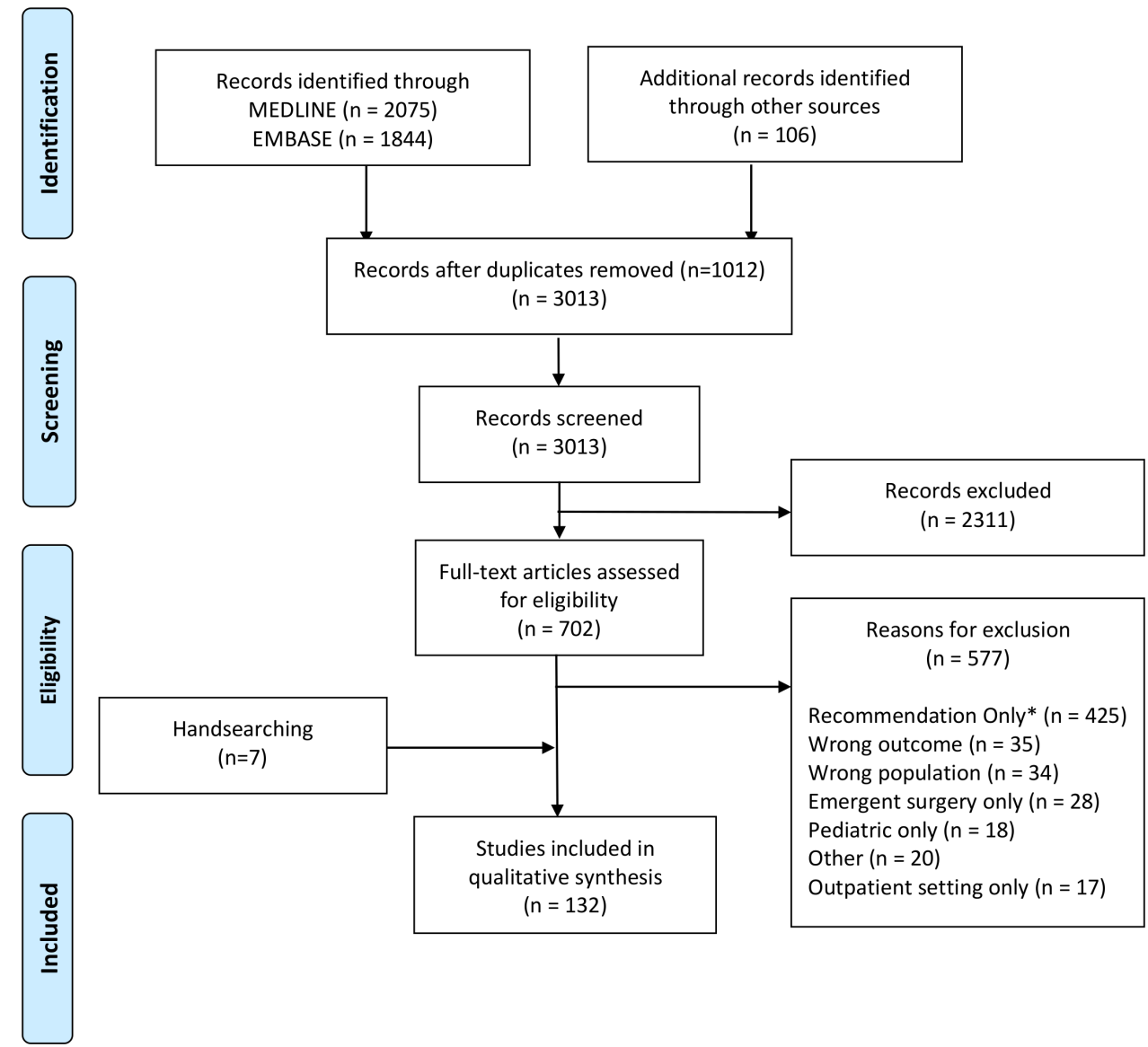

Figure 1 PRISMA flow diagram. *Includes guidelines, recommendation-based reviews, projections or estimations without mention of true changes to surgical programming. PRISMA,Preferred Reporting Items for Systematic Reviews and MetaAnalyses.

Changes to the workforce. Fourteen of the included studies describe changing the workforce into a minimum of two teams: a 'contaminated' team providing care to infected patients and a 'clean' team managing those not infected. When these teams were kept separate from one another both inside and outside of the hospital setting, surgical departments were able to continue managing the inevitable emergencies (as well as non-urgent procedures in some settings) without cross contamination during the public health emergencies. New work rotations and shift schedules were created to ensure this structure was sustainable, often with extra healthcare providers designated to replace those with exposures and to provide adequate time off to prevent burnout. This practice was only possible with wards, operating rooms and pathways (ie, corridors and elevators) that are separated under the same 'clean' and 'contaminated' designation. In the most extreme case, entire hospitals were designated for each patient group, as was done by Singapore during SARS ${ }^{16}$ and Italy during COVID-19. ${ }^{17}$

\section{Impact of reorganising surgical services}

Of the 55 studies with data relevant to this question, 42 were focused on changes in surgical volumes with six reporting changes to surgical waitlist time or composition, four underlining changes to resident and fellow involvement in surgery and two showing changes in patient pain, anxiety and depression. These recurring outcome measures are summarised below with data for all studies relevant to this question shown in (online supplemental appendix $\mathrm{C}$ ).

Changes in surgical volumes. Thirty-seven studies provided data for this outcome, with $37.8 \%(\mathrm{n}=14)$ reporting a greater than $75 \%$ reduction and $70.3 \%(\mathrm{n}=26)$ reporting a greater than $50 \%$ reductions in their overall or site specific non-urgent surgical volumes (figure 3A). Not all studies reported reductions, as one study from an oncology 'hub' hospital in Italy reported a $20 \%$ increase in their surgical volumes, likely due to more cases being diverted to their hospital during the COVID-19 pandemic. ${ }^{18}$

Changes in resident/fellow involvement in surgical activities. Four studies ${ }^{19-22}$ reported on this outcome; two surveybased case series, one resident-level case study and one study containing both survey and case $\log$ data. The reductions in surgical involvement for residents are shown by quartile in figure $3 \mathrm{~B}$.

Changes to waitlist length and composition. Five studies ${ }^{23-27}$ reported data for this outcome. One centre reported a $64 \%$ increase in length of their minor colorectal surgery waitlist ${ }^{26}$ and another centre (head and neck oncological surgery programme) reported a 500\% increase in latency 

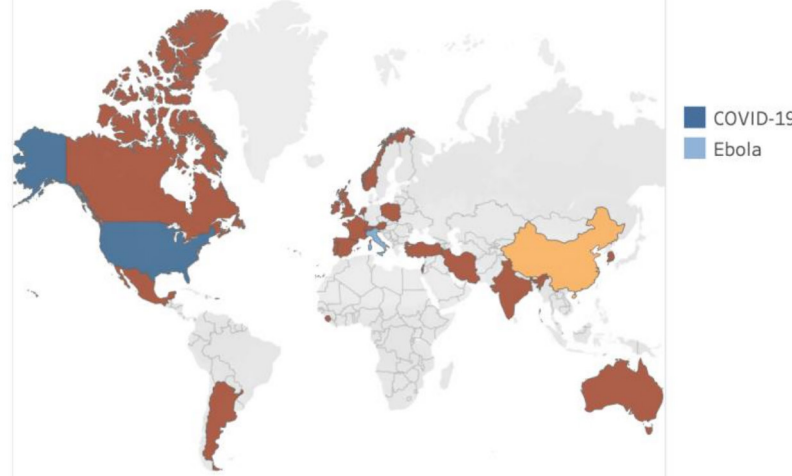

Ebola

C. Surgical Specialty

Surgical Specialty

Oncology

Orthopaedics

Neurosurgery

Opthalmology

41.00
MERS
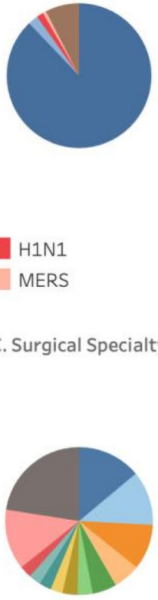

SARS

$\begin{array}{ll}\text { Otolaryngology } & \text { Plastics } \\ \text { Gl } & \text { Not specified } \\ \text { General } & \text { Other } \\ \text { Transplant } & \\ \text { Urology } & \end{array}$

Figure 2 Summary of study characteristics. (A) Country of publication, (B) public health emergency discussed and (C) surgical specialty addressed ('other' includes cardiac $(n=3)$, anaesthesia $(n=3)$, electrophysiology $(n=3)$, obstetrics and gynaecology $(n=3)$, thoracic $(n=2)$, interventional radiology $(n=1)$ and dermatology $(n=1))$.

from diagnosis to surgery. ${ }^{27}$ One study reported no waitlist deaths during the COVID-19 pandemic, ${ }^{25}$ while another saw a small decrease in the number of weekly waitlist deaths. ${ }^{24}$ A single study identified more patients leaving their renal transplantation waitlist due to mortality or clinical deterioration. ${ }^{23}$

Changes in patient pain, anxiety and depression. Two studies $^{28} 29$ reported pain, anxiety and depression among more than half of waitlist patients; $42.1 \%$ experienced anxiety and 26.3\% experienced depression (figure 3C). The leading reported cause of patient anxiety was a lack of knowledge about when their surgeries would be rescheduled. Other than a single study describing the negative financial effects of the COVID-19 pandemic, ${ }^{30}$ impacts on healthcare providers and their practices were rarely discussed.

\section{Rebuild surgical capacity}

A total of seven studies reported the experience of rebuilding surgical capacity in their departments, hospitals or systems; all studies referred to the COVID-19 pandemic. One study from China reported reopening non-urgent surgeries with close consideration of risk for imported transmission but did not provide further detail of triage or prioritisation. ${ }^{31}$ Among studies that changed their surgical triage practices, patients were prioritised for surgery based on procedure acuity or urgency (ie, risk to patients if surgery were further delayed), resource intensity and procedural complexity. Four studies ${ }^{32-35}$ noted that prior to resuming non-urgent surgeries, availability of the staff operating rooms, PPE and testing was necessary to prepare for a large and complicated surgical backlog.

\section{DISCUSSION}

This review identified over 3000 evidence sources, 132 of which were included. Approaches to reorganising surgical services varied between studies and centres, but the cancellation or postponement of non-urgent surgeries such as arthroplasty surgeries for chronic joint pain, coronary artery bypass graft surgery for asymptomatic individuals and primary gastric bypass surgery was nearly universal. ${ }^{2}$ The most frequently reported change to surgical services was modified triage criteria for surgical cases, workforce and approach to patient care. Many studies reported a decrease in surgical volumes due to public health emergencies, while a few reported the non-surgical impacts such as patient well-being or changes in healthcare utilisation beyond the surgical wards. Very few studies described their experience resuming surgical services after a public health emergency.

The varied approaches to providing surgical services during a public health emergency identified in this review illustrate that a 'one size fits all' approach does not exist. Changes to surgical services likely depends on the characteristics of specific centres and their patients. While several guidelines have been published with recommendations on how to provide surgical care during COVID19 , we chose to exclude guidelines and recommendations 
Table 2 Reorganisation of surgical services by domain

\begin{tabular}{ll}
\hline Change domain & Number of studies (\%) \\
\hline Triage or case selection & $80(74.7)$ \\
& \\
PPE & $63(58.3)$
\end{tabular}

\section{Examples of change}

1. Prioritisation of patients based on predefined levels of acuity

2. Virtual multidisciplinary meetings or tumour boards.

3. Creation of specialty-specific lists outlining surgery-eligible and ineligible ailments, often with inclusion of case-by-case category.

4. Postponement based on high-risk patient characteristics (ie, older age, multimorbidity) and expected need for ICU.

1. Hospital wide surgical mask mandate for staff and attendees.

2. Standard level of PPE outlined for all patient encounters with enhanced PPE (eg, addition of N95 or PAPR, head and shoe covering) protocol for specific procedures or care of infected patients.

3. Refresher instruction courses provided to all hospital staff.

4. Trained observer supervising all perioperative donning and doffing of PPE to ensure safety and compliance.

\begin{tabular}{|c|c|c|}
\hline Workforce & $70(64.8)$ & $\begin{array}{l}\text { 1. Separation of clinical staff into rotating 'clean' and 'dirty' teams caring } \\
\text { for exclusively for non-infected and infected patients, respectively. } \\
\text { 2. Temperature and symptom screening of staff with mandated } \\
\text { quarantine periods in cases of unprotected exposure. } \\
\text { 3. Case discussions, handover and clinical staff meetings transitioned to } \\
\text { virtual format. } \\
\text { 4. Redeployment of staff to hospital areas requiring support (eg, ICU), } \\
\text { often paired with virtual training to ensure comfortable transition. }\end{array}$ \\
\hline Patient care & $95(88.0)$ & $\begin{array}{l}\text { 1. Complete cancellation or transition to telemedicine for all non-urgent } \\
\text { and routine perioperative clinical visits. } \\
\text { 2. Patient temperature, symptom and travel history screening before } \\
\text { entry to clinic (relevant for urgent surgical patients). } \\
\text { 3. Preference for endovascular or minimally invasive surgical approaches } \\
\text { when possible, use of conservative care when possible (oncology). } \\
\text { 4. Restrictions on number of accompanying persons or visitors (often } \\
\text { zero with some allowing maximum of 1). }\end{array}$ \\
\hline Resident/fellow education & $35(32.4)$ & $\begin{array}{l}\text { 1. Changes to resident/fellow team structure and rotation schedules to } \\
\text { ensure continued coverage of department and maximise resident/ } \\
\text { fellow safety. } \\
\text { 2. Redeployment of residents to non-specialty areas requiring clinical } \\
\text { support. } \\
\text { 3. Curriculum and conferences shifted to online format to allow } \\
\text { continued e-learning for off-duty trainees. } \\
\text { 4. Trainees involvement in surgical care of infected persons ceased or } \\
\text { altered (eg, only admitted to OR during low-risk/non-aerosolising } \\
\text { procedures). }\end{array}$ \\
\hline Environment & $70(64.8)$ & $\begin{array}{l}\text { 1. Dedication of wards (hallways and elevators), ORs or entire hospitals } \\
\text { to treat for only those infected or not infected. } \\
\text { 2. Use of negative-pressure OR when possible. } \\
\text { 3. Transformation of surgical wards, ORs and outpatient clinics into } \\
\text { patient care areas to increase surge capacity. } \\
\text { 4. Double occupancy patient rooms reduced to single occupancy or } \\
\text { physical measures (eg, cubicles, distanced waiting room chairs) } \\
\text { implemented. }\end{array}$ \\
\hline
\end{tabular}

ICU, intensive care unit; OR, operating room; PAPR, powered air purifying respirator; PPE, personal protective equipment.

from this review for two reasons: (1) a high-quality review of surgical recommendations for the response to COVID-19 was published by one of the authors just prior to this study ${ }^{10}$ and (2) because there is abundant evidence suggesting guidelines and recommendations for practice are frequently not implemented into clinical practice. ${ }^{36-42}$ Some of the guideline recommendations in the review by Søreide $e t a l^{10}$ were implemented within the included studies in the present review, such as creating areas within-hospital for 'clean' and 'contaminated' cases and workforce redeployment to critical care. However, other recommendations were infrequently noted, such as the dedicated use of isolated, negative pressure ORs for patients with COVID-19. These resource intensive 


\section{A. Reductions in Surgical Activity}

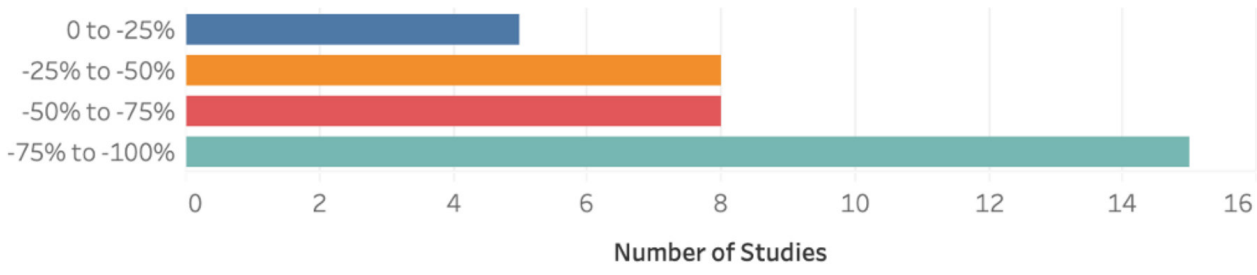

B. Reductions in Resident/Fellow Surgical Involvement

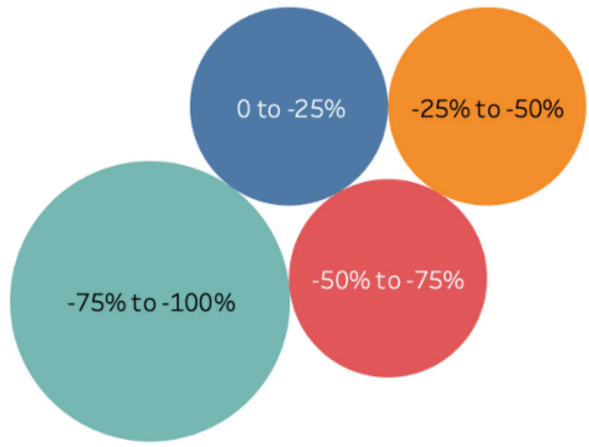

C. Surgical Waitlist Patient Experience

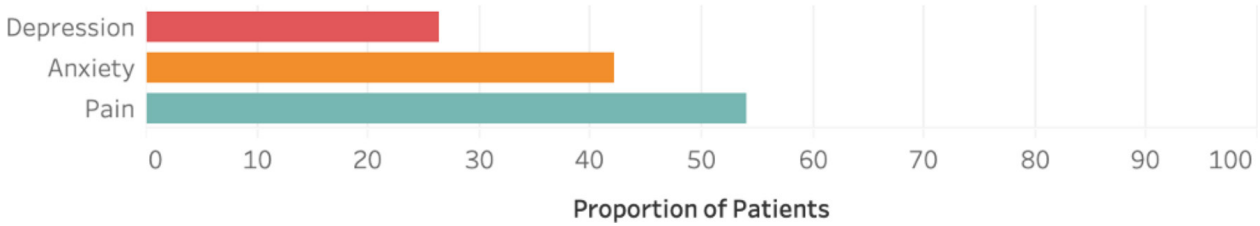

Figure 3 Summary of leading impacts of changes to surgical programming. A summary of the impacts of alterations to surgical services during public health emergencies on: (A) overall surgical activity ( $n=37$ studies), (B) resident and fellow involvement in surgery ( $n=5$ studies) where circle size represents the number of studies contributing to that quartile and (C) patient experience ( $\mathrm{n}=2$ studies).

practices may not have been attainable under the pressures of managing public health emergencies and may not be feasible in low-resource settings.

Changes to surgical services, such as cancelling or postponing non-urgent surgeries may be necessary to manage public health emergencies to reduce the risk of contamination and increase capacity within hospitals. However, the impact of these changes remains poorly understood. Many studies reported decreases in surgical volumes, but few other variables were explored with regards to the impact on patients, providers and healthcare systems. Five studies examined the impact of changes to surgical services among physicians and trainees and found that training was compromised in some specialties. ${ }^{19-22}$ The finding that medical training was compromised is particularly important for understanding the downstream and long-term repercussions of the response to public health emergencies; decreases in surgical volumes and clinical hours for trainees could have negative and unintended effects on the future quality and safety of patient care. ${ }^{43}$ Notably, the impacts of public health emergencies on medical training and education were almost exclusively evaluated for residents and fellows, failing to consider the limited access that current medical undergraduate students continue to encounter when trying to explore surgical specialties. This is unlikely to affect the quality of patient care but may present later in the form of decreased career satisfaction and engagement, both of which have been associated with burnout. ${ }^{44}$ Studies examining the effects of surgical service alterations on patients noted negative effects on mental health outcomes, ${ }^{28}{ }^{29}$ pain $^{28}$ and an increased incidence of death among surgical patients. ${ }^{23} 2445$

Very few studies described specific actions undertaken to rebuild and resume prepublic health emergencies surgical capacity. This may be due to the fact that most included studies examined the ongoing COVID-19 pandemic, or because few places have implemented specific plans to date. Included studies did describe consideration of system-level factors like availability of PPE and ORs. However, more patient-centric considerations such as organising childcare and requesting time away from their job during a pandemic are needed. Additionally, research suggesting that surgical capacity can be rebuilt with sufficient PPE and OR space may be falling victim to the lack of identified evidence exploring the 
well-being of the surgical workforce. Resolving surgical backlogs by increasing available resources relies on the high functioning of a workforce of surgeons and allied practitioners not overtaken by burnout and stress, something that has not yet been borne out in the COVID-19 research. In other specialties involved with the care of surgical patients, moral distress has seen a marked increase making it reasonable to believe these same emotional impacts will be felt by members or surgical teams globally. Patient perspectives will also play a role in the rebuild; one study reported $14 \%$ of surgical patients initiated the cancellation of their surgery, ${ }^{28}$ which suggests patient readiness for surgery during and post COVID-19 should be considered. For evidence to inform policy, additional research is needed to understand the impacts of different approaches for resuming surgical services.

This study is, to our knowledge, the first comprehensive scoping review of evidence around reallocation of surgical services during public health emergencies. While this study has several strengths, including a comprehensive search of academic and grey literature sources, and a mix of inductive and deductive data abstraction approaches, there are some limitations that should be considered when interpreting our findings. We modified the Joanna Briggs methodology for scoping reviews, ${ }^{5}$ according to the WHO and Cochrane's guidance on conducting rapid reviews, ${ }^{78}$ with the intent of balancing rigour with a timely and policy responsive review of the literature. Also, given that the evidence around the COVID-19 pandemic is growing at an unprecedented rate, we are aware that additional studies have been published since we ran our search strategy, especially around resuming surgical services. In order to mitigate this limitation, an ongoing effort to pivot this study into a living review is underway to ensure the data presented is up to date. This will involve rerunning the MEDLINE, Embase and grey literature search strategies every 2 months in order to incorporate new evidence into the existing manuscript. Notably, this review did not identify evidence from any low-income or middle-income countries who may face unique challenges during a pandemic compared with high-income countries described in our review. It is also likely that during the global pandemic, many healthcare institutions have been focused on coping with COVID-19 instead of publishing their experiences; we hope more organisations will add their experience to the literature.

In conclusion, we report early evidence of the operational changes that have occurred internationally in response to public health emergencies that could inform the ongoing response to COVID-19 and future public health emergencies. This study identified a gap in our understanding of the impact of these changes on patients, providers and the healthcare system that should be the focus of research moving forward to provide an evidencebased approach to managing surgical patients in future public health emergencies.

\section{Original protocol for the study}

The original unpublished protocol for this study is included as a supplementary file (online supplemental appendix D).

Author affiliations

${ }^{1}$ Community Health Sciences, University of Calgary, Calgary, Alberta, Canada

${ }^{2}$ Department of Critical Care Medicine, University of Calgary Cumming School of

Medicine, Calgary, Alberta, Canada

${ }^{3}$ Alberta Health Services, Edmonton, Alberta, Canada

${ }^{4}$ University of Calgary, Calgary, Alberta, Canada

${ }^{5}$ Department of Surgery, University of Alberta, Edmonton, Alberta, Canada

${ }^{6}$ Surgery Strategic Clinical Network, Alberta Health Services, Edmonton, Alberta, Canada

${ }^{7}$ Department of Pediatrics, University of Calgary Cumming School of Medicine, Calgary, Alberta, Canada

${ }^{8}$ Community Health Sciences, Surgery \& Oncology, University of Calgary, Calgary, Alberta, Canada

\section{Twitter Khara Sauro @kharasauro}

Contributors $\mathrm{CO}$ contributed to the design and conceptualisation of the review, analysis and interpretations of the data, and drafting and revising the manuscript; $\mathrm{JN}-\mathrm{K}$ and AK-R contributed to the design of the review, interpretation of the data, providing feedback on the manuscript and gave approval of the final version of the manuscript; JD, JW, JR and MB contributed to the interpretation of the data, providing feedback on the manuscript and gave approval of the final version of the manuscript; KS contributed to the design and conceptualisation of the review, analysis and interpretations of the data, providing feedback on the manuscript and gave approval of the final version of the manuscript.

Funding The authors have not declared a specific grant for this research from any funding agency in the public, commercial or not-for-profit sectors.

Map disclaimer The depiction of boundaries on the map(s) in this article does not imply the expression of any opinion whatsoever on the part of BMJ (or any member of its group) concerning the legal status of any country, territory, jurisdiction or area or of its authorities. The map(s) are provided without any warranty of any kind, either express or implied.

Competing interests None declared.

Patient consent for publication Not required.

Provenance and peer review Not commissioned; externally peer reviewed.

Data availability statement Data are available on reasonable request. All data presented have been previously published. Our data will be made available on reasonable request to the corresponding author.

Supplemental material This content has been supplied by the author(s). It has not been vetted by BMJ Publishing Group Limited (BMJ) and may not have been peer-reviewed. Any opinions or recommendations discussed are solely those of the author(s) and are not endorsed by BMJ. BMJ disclaims all liability and responsibility arising from any reliance placed on the content. Where the content includes any translated material, BMJ does not warrant the accuracy and reliability of the translations (including but not limited to local regulations, clinical guidelines, terminology, drug names and drug dosages), and is not responsible for any error and/or omissions arising from translation and adaptation or otherwise.

Open access This is an open access article distributed in accordance with the Creative Commons Attribution Non Commercial (CC BY-NC 4.0) license, which permits others to distribute, remix, adapt, build upon this work non-commercially, and license their derivative works on different terms, provided the original work is properly cited, appropriate credit is given, any changes made indicated, and the use is non-commercial. See: http://creativecommons.org/licenses/by-nc/4.0/.

ORCID iD

Khara Sauro http://orcid.org/0000-0002-7658-4351

\section{REFERENCES}

1 Johns Hopkins Center for Systems of Science and Engineering (CSSE). COVID-19 Dashboard. Available: http://coronavirus.jhu.edu/ map.html [Accessed 8 Jan 2020]. 
2 American College of Surgeons. COVID-19: recommendations for management of elective surgical procedures 2020, 2020. Available: https://www.facs.org/-/media/files/covid19/recommendations_for_ management_of_elective_surgical_procedures.ashx [Accessed 2 Jul 2020].

3 Shared Health Manitoba. COVID-19: the new normal. Available: https://sharedhealthmb.ca/files/covid-19-memo-the-new-normal.pdf [Accessed 8 May 2020]

4 COVIDSurg Collaborative. Elective surgery cancellations due to the COVID-19 pandemic: global predictive modelling to inform surgical recovery plans. Br J Surg 2020;107:1440-9.

5 Peters MDJ, Godfrey CM, Mclnerney P. Chapter 11: Scoping Reviews. In: Aromataris E, Munn Z, eds. Joanna Briggs Institute Reviewer's Manual. The Joanna Briggs Institute, 2017.

6 Tricco AC, Lillie E, Zarin W, et al. PRISMA extension for scoping reviews (PRISMA-ScR): checklist and explanation. Ann Intern Med 2018;169:467-73.

7 Tricco AC, Langlois EV, Straus SE, eds. Rapid reviews to strengthen health policy and systems: a practical guide. Geneva: World Health Organization, 2017.

8 Garritty C, Gartlehner G, Kamel C. Cochrane rapid reviews. interim guidance from the Cochrane rapid review methods group, 2020.

9 Godin K, Stapleton J, Kirkpatrick SI, et al. Applying systematic review search methods to the grey literature: a case study examining guidelines for school-based breakfast programs in Canada. Syst Rev 2015;4:138.

10 Søreide K, Hallet J, Matthews JB, et al. Immediate and long-term impact of the COVID-19 pandemic on delivery of surgical services. Br J Surg 2020;107:1250-61.

11 Munn Z, Peters MDJ, Stern C, et al. Systematic review or scoping review? guidance for authors when choosing between a systematic or scoping review approach. BMC Med Res Methodol 2018;18:143.

12 Arksey H, O'Malley L. Scoping studies: towards a methodological framework. Int J Soc Res Methodol 2005;8:19-32.

13 Álvarez Gallego M, Gortázar de Las Casas S, Pascual Migueláñez I, et al. SARS-CoV-2 pandemic on the activity and professionals of a general surgery and digestive surgery service in a tertiary hospital. Cir Esp 2020;98:320-7.

14 Wang X, Wang Z, Yao C. Management of ophthalmic perioperative period during 2019 novel coronavirus disease outbreak. Chin J Exp Ophthalmol 2020;38:200-3.

15 Zhen L, Lin T, Zhao ML, et al. [Management strategy for the resumption of regular diagnosis and treatment in gastrointestinal surgery department during the outbreak of coronavirus disease 2019 (COVID-19)]. Zhonghua Wei Chang Wai Ke Za Zhi 2020;23:321-6.

16 Chee VWT, Khoo ML-C, Lee SF, et al. Infection control measures for operative procedures in severe acute respiratory Syndrome-related patients. Anesthesiology 2004;100:1394-8.

17 Fontanella MM, Saraceno G, Lei T, et al. Neurosurgical activity during COVID-19 pandemic: an experts' opinion from China, South Korea, Italy, the USA, Colombia, and the UK. J Neurosurg Sci 2020;64.

18 Vicini E, Galimberti V, Naninato P, et al. COVID-19: The European institute of oncology as a "hub" centre for breast cancer surgery during the pandemic in Milan (Lombardy region, northern Italy) - A screenshot of the first month. Eur J Surg Oncol 2020;46:1180-1.

19 Amparore D, Claps F, Cacciamani GE, et al. Impact of the COVID-19 pandemic on urology residency training in Italy. Minerva Urol Nefrol 2020;72.

20 Cai Y, Jiam NT, Wai KC, et al. Otolaryngology Resident Practices and Perceptions in the Initial Phase of the U.S. COVID -19 Pandemic. Laryngoscope 2020;130:2550-7.

21 Givi B, Moore MG, Bewley AF, et al. Advanced head and neck surgery training during the COVID-19 pandemic. Head Neck 2020;42:1411-7.

22 Hemingway JF, Singh N, Starnes BW. Emerging practice patterns in vascular surgery during the COVID-19 pandemic. J Vasc Surg 2020;72:396-402.
23 de Vries APJ, Alwayn IPJ, Hoek RAS, et al. Immediate impact of COVID-19 on transplant activity in the Netherlands. Transpl Immunol 2020;61:101304.

24 Domínguez-Gil B, Coll E, Fernández-Ruiz M, et al. COVID-19 in Spain: transplantation in the midst of the pandemic. Am J Transplant 2020;20:2593-8.

25 Lauterio A, De Carlis R, Belli L, et al. How to guarantee liver transplantation in the North of Italy during the COVID-19 pandemic: a sound transplant protection strategy. Transp/ Int 2020;33:969-70.

26 Bradford IMJ. Tales from the frontline: the colorectal battle against SARS. Colorectal Dis 2004;6:121-3.

27 Lee AKF, Cho RHW, Lau EHL, et al. Mitigation of head and neck cancer service disruption during COVID-19 in Hong Kong through telehealth and multi-institutional collaboration. Head Neck 2020;42:1454-9.

28 Brown TS, Bedard NA, Rojas EO, et al. The Effect of the COVID-19 Pandemic on Electively Scheduled Hip and Knee Arthroplasty Patients in the United States. J Arthroplasty 2020;35:S49-55.

29 IYP W, KHY W, CSH N. Evaluation of the emotional status of patients on a waiting list for thoracic surgery during the outbreak of Severe Acute Respiratory Syndrome(SARS). Stress and Health 2004;20:209-12.

30 Vaccaro AR, Getz CL, Cohen BE, et al. Practice management during the COVID-19 pandemic. J Am Acad Orthop Surg 2020;28:464-70.

31 Shen Y, Cui Y, Li N, et al. Emergency responses to Covid-19 outbreak: experiences and lessons from a general Hospital in Nanjing, China. Cardiovasc Intervent Radiol 2020;43:810-9.

32 Busin M, Yu AC, Ponzin D. Coping with COVID-19: an Italian perspective on corneal surgery and eye banking in the time of a pandemic and beyond. Ophthalmology 2020;127:e68-9.

33 Pelt CE, Campbell KL, Gililland JM, et al. The rapid response to the COVID-19 pandemic by the arthroplasty divisions at two academic referral centers. J Arthroplasty 2020;35:S10-14.

34 Walker JP. Resuming elective surgery at UTMB predicated on patient and staff well-being, 2020.

$35 \mathrm{BC}$ Ministry of Health. A commitment to surgical renewal in bc. Canada, 2020.

36 Ben-Menachem E, French JA. Guidelines?Are They Useful? Epilepsia 2006;47:62-4.

37 Cabana MD, Rand CS, Powe NR, et al. Why don't physicians follow clinical practice guidelines? A framework for improvement. JAMA 1999;282:1458-65.

38 Farquhar CM, Kofa EW, Slutsky JR. Clinicians' attitudes to clinical practice guidelines: a systematic review. Med J Aust 2002;177:502-6.

39 Grimshaw JM, Russell IT. Effect of clinical guidelines on medical practice: a systematic review of rigorous evaluations. The Lancet 1993;342:1317-22

40 Grimshaw JM, Thomas RE, MacLennan G, et al. Effectiveness and efficiency of guideline dissemination and implementation strategies. Health Technol Assess 2004;8:1-72.

41 Lomas J, Anderson GM, Domnick-Pierre K, et al. Do practice guidelines guide practice? the effect of a consensus statement on the practice of physicians. N Engl J Med 1989;321:1306-11.

42 Lugtenberg M, Burgers JS, Westert GP. Effects of evidence-based clinical practice guidelines on quality of care: a systematic review. Qual Saf Health Care 2009;18:385-92.

43 Ahmed N, Devitt KS, Keshet I, et al. A systematic review of the effects of resident duty hour restrictions in surgery: impact on resident wellness, training, and patient outcomes. Ann Surg 2014;259:1041-53.

44 Rao S, Ferris TG, Hidrue MK, et al. Physician burnout, engagement and career satisfaction in a large academic medical practice. Clin Med Res 2020;18:3-10.

45 D'Apolito R, Faraldi M, Ottaiano I, et al. Disruption of arthroplasty practice in an orthopedic center in northern Italy during the coronavirus disease 2019 pandemic. J Arthroplasty 2020;35:S6-9. 